\title{
QUALIDADE AMBIENTAL DO SOLO SOB A ÁREA DO ATERRO CONTROLADO DO MUNICÍPIO DE CORRENTE-PI
}

\author{
Environmental quality soil in the landfill area controlled in Corrente - PI
}

\section{Juliana Vogado Coelho ${ }^{1}$; \\ Tancio Gutier Ailan Costa ${ }^{2}$; \\ Bruna de Freitas Iwata ${ }^{3}$.}

RESUMO: A contaminação do solo atua como um dos principais problemas no que se refere ao destino inadequado dos resíduos sólidos. Considerando esse processo, o estudo buscou verificar a qualidade ambiental do solo da área sob o aterro controlado do município de Corrente-PI. Através de visita in loco buscou-se identificar os possíveis meios de contaminação do solo, avaliando a disposição dos resíduos, a topografia do local e verificação das técnicas de proteção ao solo/ambiente. No que se refere à disposição dos resíduos sólidos do município identifica-se como irregular, possivelmente provocando a contaminação do solo por não executar o tratamento prévio adequado dos resíduos. Tendo em destaque o aspecto topográfico da área no qual observou-se o relevo suave, de modo ao qual possibilita minimizar o processo de erosão. Diante dos fatores que se encontram irregular na área sob o aterro controlado alguns visam à utilização de um planejamento integral na gestão dos resíduos sólidos no qual se encontram em estado de deficiência na destinação final de tais resíduos, visando promover como subsidio para minimizar possíveis meios de contaminação do solo. A identificação e monitoramento dos resíduos que são depositados na área podem ser realizados de maneira que estabeleça um controle que possa intervir na contaminação do solo na área, mediante diagnóstico detalhado visando os riscos que os resíduos podem causar e possivelmente uma investigação confirmatória dos possíveis fatores que alteram a qualidade do solo.

PALAVRAS-CHAVE: Resíduos Sólidos; Impacto Ambiental; Qualidade Ambiental.

ABSTRACT: Soil contamination acts as a major problem that stands out with regard to the inadequate disposal of solid waste. Thus, the study looked at the environmental quality of the soil

\footnotetext{
${ }^{1}$ Acadêmica do curso de Tecnologia em Gestão Ambiental- Instituto Federal de Educação, Ciência e Tecnologia do Piauí - IFPI- Campus Corrente. E-mail: vogado.juliana@hotmail.com.

${ }^{2}$ Acadêmico do curso de Tecnologia em Gestão Ambiental- Instituto Federal de Educação, Ciência e Tecnologia do Piauí - IFPI- Campus Corrente. E-mail: gutierailan@gmail.com.

${ }^{3}$ Professora do curso de Tecnologia em Gestão Ambiental- Instituto Federal de Educação, Ciência e Tecnologia do Piauí - IFPI- Campus Corrente. E-mail: iwata@ifpi.edu.br.
} 
of the area under the landfill in the municipality of Corrente-PI. Through business in loco sought to identify potential soil contamination means of evaluating the disposal of waste, local topography and verification of protection techniques to the ground/environment. As regards the disposal of municipal solid waste is identified as irregular possibly causing contamination of the soil not to perform the appropriate pretreatment of the residues. In highlight the topographical feature area in which there was a gentle slope, so which permits minimizing soil erosion process and possibly facilitate the maintenance of components of the drainage system, prevents the growth of possible contaminating factors from soil. On the factors that are irregular in the area under the landfill controlled some aimed at the use of a comprehensive planning in solid waste management in which they are in a state of deficiency in the final disposal of such waste, to promote as a subsidy to minimize possible means of soil contamination. The identification and monitoring of waste that is deposited in the area can be performed in view of a control that can intervene in the soil contamination in the area, through detailed diagnosis targeting the risks that waste can cause and possibly a confirmatory investigation of possible factors that alter soil quality.

KEYWORDS: Solid Waste; Environmental Impact; Environmental Quality. 


\section{INTRODUÇÃO}

A perda de qualidade do solo é uma das principais formas de promoção de danos ao ambiente em geral, considerando a dependência de outros recursos em relação ao mesmo. Em ambientes urbanos o processo de perda de qualidade do solo está fortemente vinculado à disposição irregular de resíduos acarretando na contaminação do solo, um dos mais graves problemas ambientais. Quando dispostos de forma inadequada, os resíduos sólidos podem causar a poluição da água, do ar e do solo, além de criar ambiente propicio para a proliferação de macro e micro vetores causadores de doenças, com consequências significativas para o meio ambiente e para a qualidade de vida da população (Besen, 2011).

Os aterros recebem resíduos de diversas origens, de baixa periculosidade, como resíduo domiciliar e comercial até aqueles altamente poluentes, como industrial e hospitalar, liberando, portanto, substâncias orgânicas e inorgânicas que são carreadas pela água para os horizontes profundos do solo, comprometendo a qualidade e o uso desse recurso (Sisinno e Moreira, 1996).

Nas áreas urbanas os resíduos depositados sobre a superfície, sem o devido tratamento, causam possíveis meios de contaminação do solo, ocasionados pelo lançamento de detritos e substancias química, que constitui problemas ambientais que necessitam de atenção da comunidade e do município para efetivas técnicas de minimização dos mesmos.

As áreas destinadas a receber toneladas de resíduos sólidos sem infra-estrutura adequada, têm seus usos futuros comprometidos e são responsáveis pela degradação ambiental das regiões sob sua influência. Os aterros controlados são mais eficientes que os lixões conforme a Funasa (2006), destaca que o aterro controlado é uma técnica de disposição de resíduos sólidos no solo, visando à minimização dos impactos ambientais. $O$ aterro controlado utiliza alguns métodos de posição que reduz poluição, porem de forma controlada, podendo comprometer a qualidade do solo.

A contaminação do solo tem-se tornado uma das preocupações ambientais, tornando-se problema quando há uma fonte de contaminação; vias de transferência de poluentes que viabilizam o alargamento da área contaminada e há indivíduos e bens ameaçados por essa contaminação.

Conforme descrito por Melo (2000), o conhecimento das áreas destinadas à disposição de resíduos, bem como o monitoramento dos resíduos aterrados, dos líquidos e gases gerados, torna-se necessário para que sejam adotadas soluções viáveis e sustentáveis tanto do ponto de vista ambiental e sanitário como socioeconômico para evitar os possíveis impactos ambientais provocados pela disposição de resíduos. 
Existe uma elevada demanda de modelo para adequação das áreas de aterro de modo a prevenir contra possíveis danos ambientais, principalmente em se tratando das alterações que podem ocorrer no solo. Diante disso, o estudo buscou verificar a qualidade ambiental do solo da área sob o aterro controlado do município de Corrente, Piauí.

\section{MATERIAL E MÉTODOS}

\section{Área de Estudo}

O estudo foi realizado no município de Corrente, que localiza-se no sul do estado do Piauí (10²6'36"; 4509'44"), a $864 \mathrm{~km}$ da capital do estado (Figura 1). Com altitude de 438 metros e uma população de 25.408 habitantes de acordo com o último censo do IBGE (2010). O município localiza-se no bioma Cerrado, com clima tropical semiúmido quente e período chuvoso concentrado principalmente entre os meses de janeiro a abril.

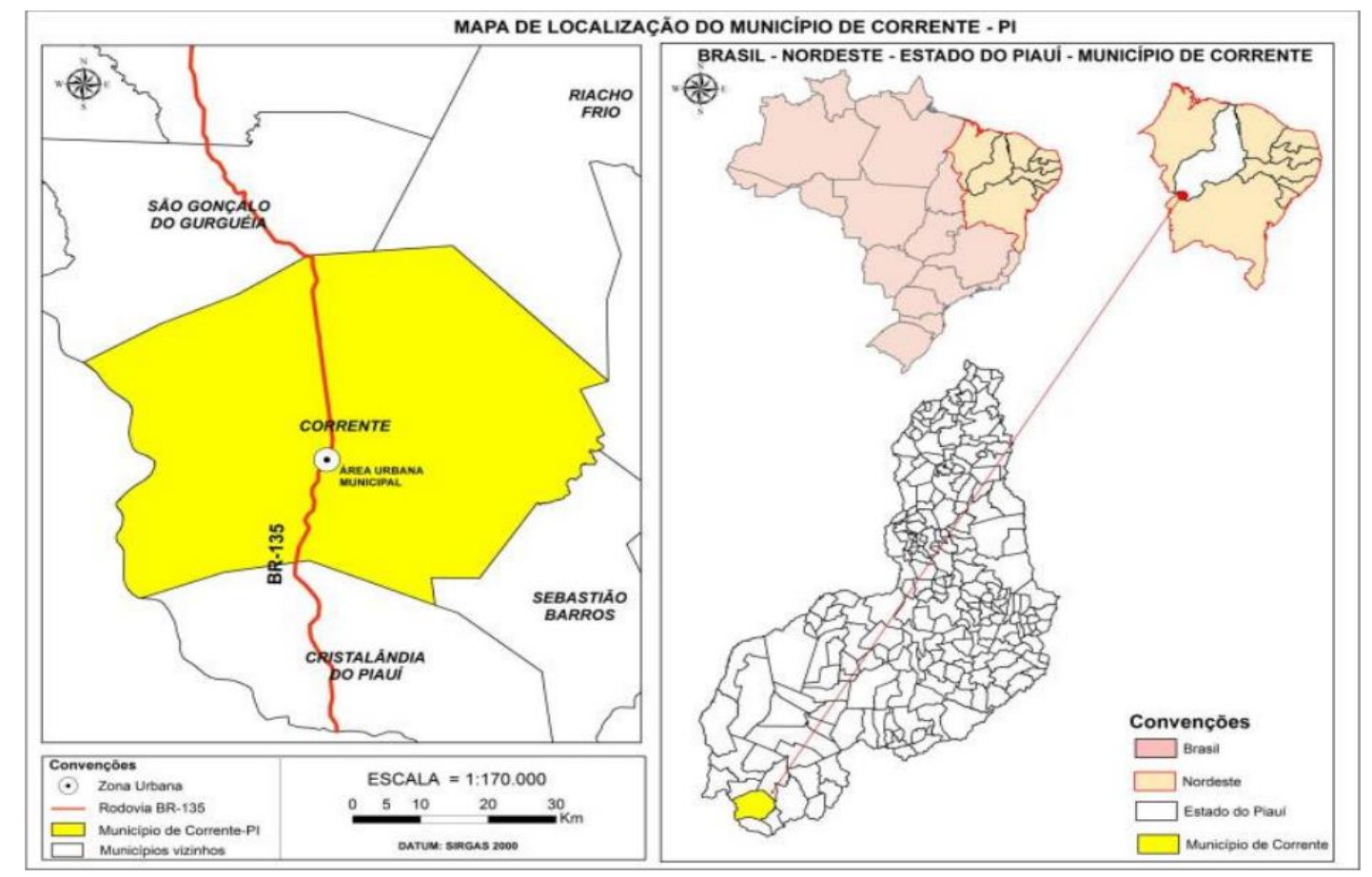

Figura 1: Localização do município de Corrente. Fonte: Antônio Celso Leite Sousa, 2015.

O local de disposição final dos resíduos sólidos urbanos e rurais tem apenas dois (2) anos de uso, sendo licenciado pela Prefeitura Municipal, que fica localizado a $10 \mathrm{~km}$ da zona urbano da cidade de Corrente, Piauí (Figura 2). Foi construído em uma propriedade da Prefeitura Municipal, situado às margens da PI 225, estrada pavimentada que liga o município de Corrente ao de Parnaguá. A área tem aproximadamente cinco (5) ha de extensão e localiza-se conforme coordenadas geográficas da área: latitude sul 10²7’47” e longitude oeste 4503’52” (PMC, 2014). 


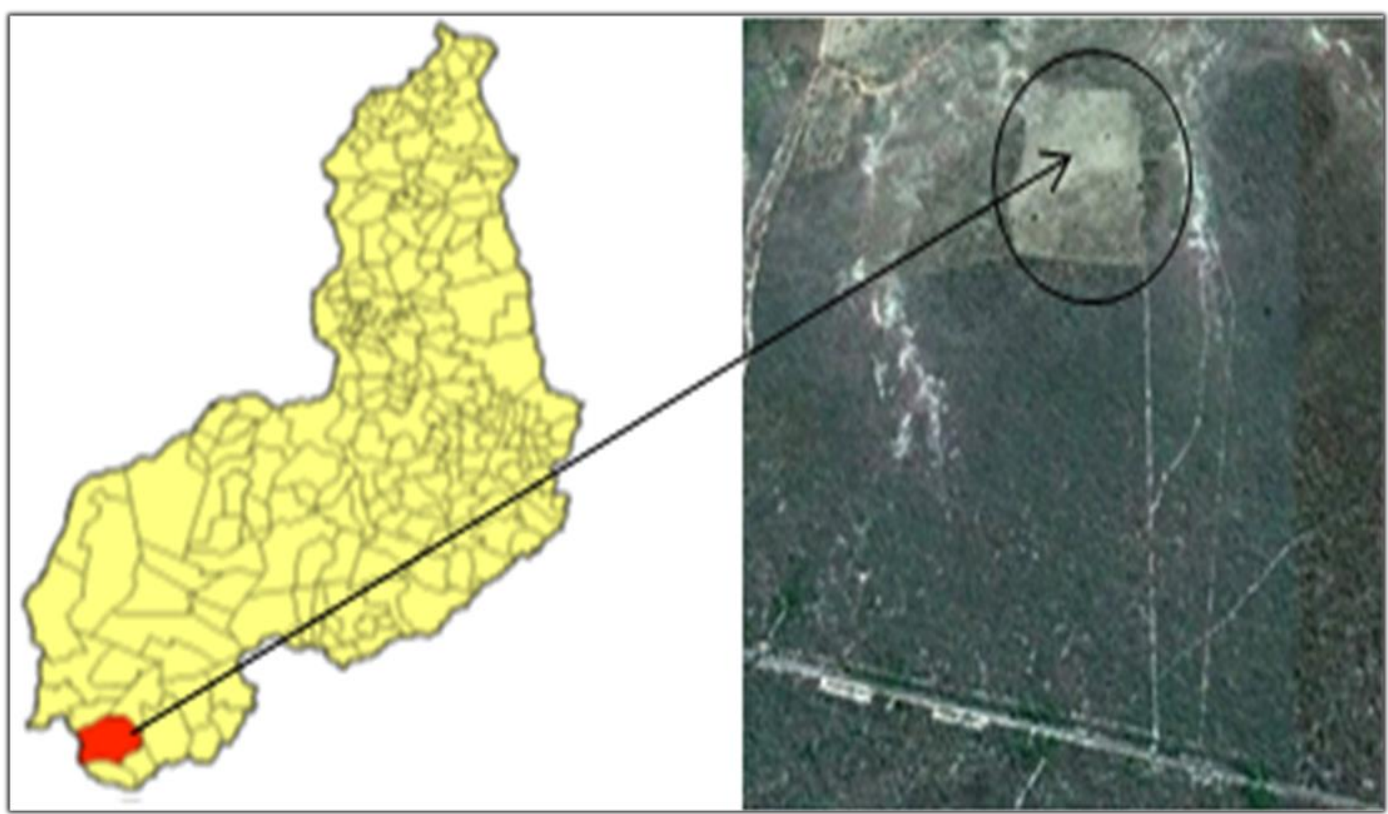

Figura 2. Área do aterro controlado do município de Corrente-Piauí, com destaque no mapa estadual. Fonte: Google Earth, 2015.

\section{Aspectos Metodológicos}

O estudo realizou visitação in loco no mês de abril de 2015, com vista a fazer uma caracterização ambiental da área, observar as possibilidades de contaminação do solo, os principais agentes responsáveis, os procedimentos de destinação e manejo dos resíduos, ações de proteção contra contato direto de resíduos de maior facilidade de contaminação ou dispersão, fatores de conservação ambiental, ações de alteração ou degradação do solo. Além disso, por meio das visitas foram levantados possíveis processos de erosão.

\section{RESULTADOS E DISCUSSÃO}

Relativo à disposição dos resíduos sólidos do município, verificou-se consistentes irregularidades visto que não ocorre tratamento prévio dos resíduos e pela disposição direta sobre o solo. Além disso, não há segregação dos tipos de resíduos depositados, logo não há qualquer medida de proteção relativa aos resíduos de maior grau de contaminação, exceto pelos resíduos sólidos de saúde que são incinerados no local (Figura 3). De acordo com Monteiro et al., (2001) a qualidade da operação de coleta e transporte de lixo depende da forma adequada do seu acondicionamento, armazenamento e da disposição dos recipientes no local, dia e horários estabelecidos pelo órgão de limpeza urbana para a coleta. Relacionando à importância que o acondicionamento adequado do lixo pode-se ressaltar a questão da atratividade que os resíduos 
podem exercer na questão social e ambiental, visando minimiza o impacto visual e olfativo, a proliferação de vetores e evita possíveis riscos de acidente.

Castilhos Junior (2003) e Paris (2007) identificam no caso dos lixões a céu aberto, como uma forma de deposição desordenada, sem a devida compactação do lixo e sem qualquer cobertura, o que acentua os problemas de contaminação do solo, do lençol freático e a proliferação de macro e micro vetores.

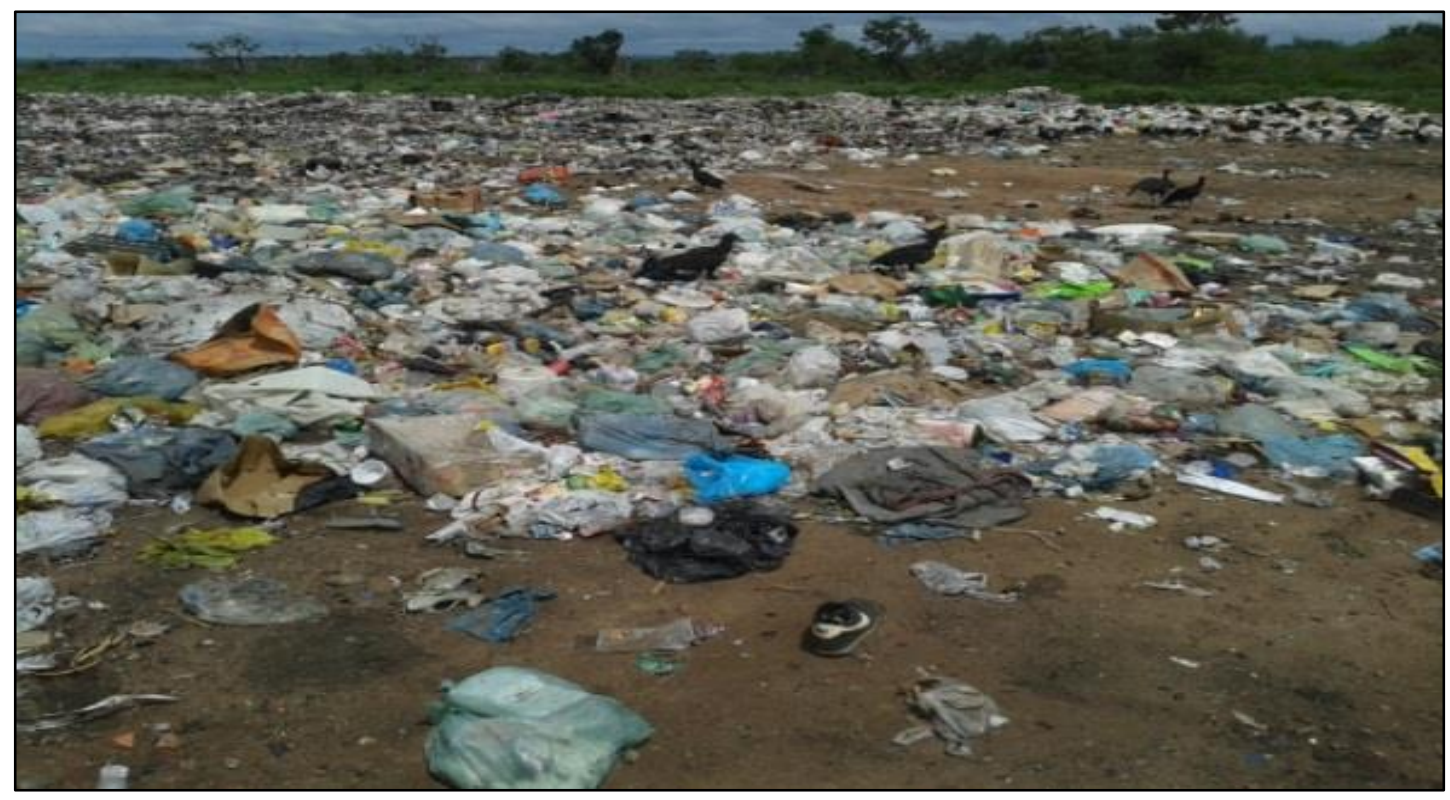

Figura 3: Disposição dos resíduos sólidos diretamente sobre o solo. Levantamento e campo, 2015.

Tendo em destaque o aspecto topográfico da área no qual observa o relevo suave, (Figura 4) de modo ao qual possibilita minimizar o processo de erosão do solo e que possivelmente facilitaria na manutenção dos componentes do sistema de drenagem, impedindo o incremento de possíveis fatores de contaminação do solo. Uma vez que para Sobrinho (2000) quando a intensidade da precipitação supera a velocidade de infiltração ocorre o acúmulo de água sobre a superfície do terreno, ficando empoçada nas depressões. $\mathrm{O}$ acumulo de água ocasionada em consequência da declividade do terreno pode atrair vetores devido ao mau odor ocasionado em decorrência da decomposição dos resíduos e possivelmente infiltra-se no solo podendo causar alteração na água subterrânea entrando em contado direto com o lençol freático. 


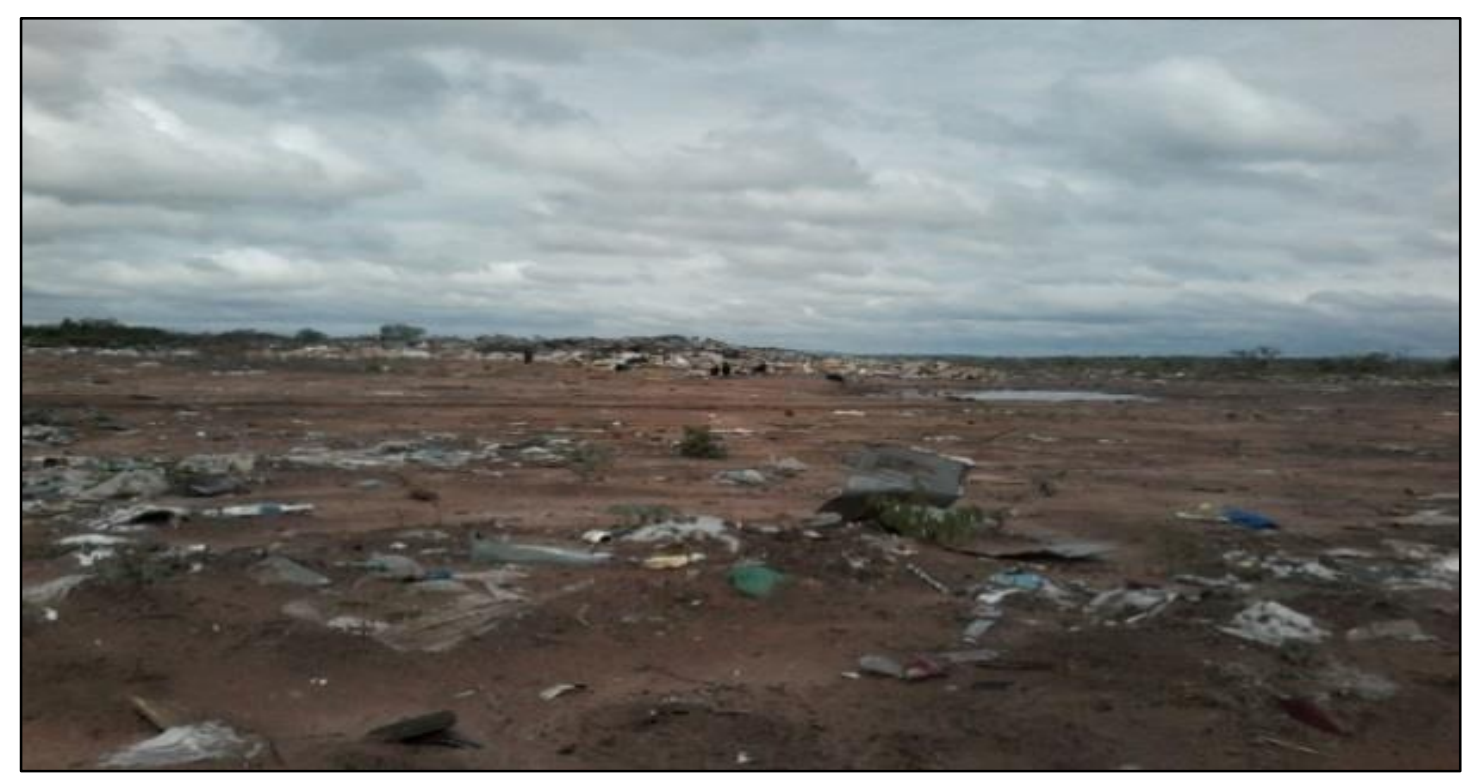

Figura 4: Aspecto topográfico da área sob o aterro controlado. Levantamento de campo, 2015.

No entanto, destaca-se que embora um relevo suave, com a disposição dos resíduos diretamente sobre o solo pode provocar alteração ao ambiente (Figura 5), promovendo alterações do microrelevo, assim, podendo facilitar os processos erosivos. Referindo- se a lixiviação que pode ser causado pelo acumulo dos resíduos após a ocorrência de um índice elevando de pluviosidade, possivelmente ocorrerá à percolação sob o solo. Teixeira (2008) após avaliar a contaminação do solo da área sob deposito de resíduos sólidos urbanos afirma que apercolação do lixiviado através do solo causa a contaminação deste e pode causar a das águas subterrâneas e superficiais. Fernández Viña (2000) afirma que o percolado aparece em consequência, principalmente, da infiltração de água na massa dos resíduos. 


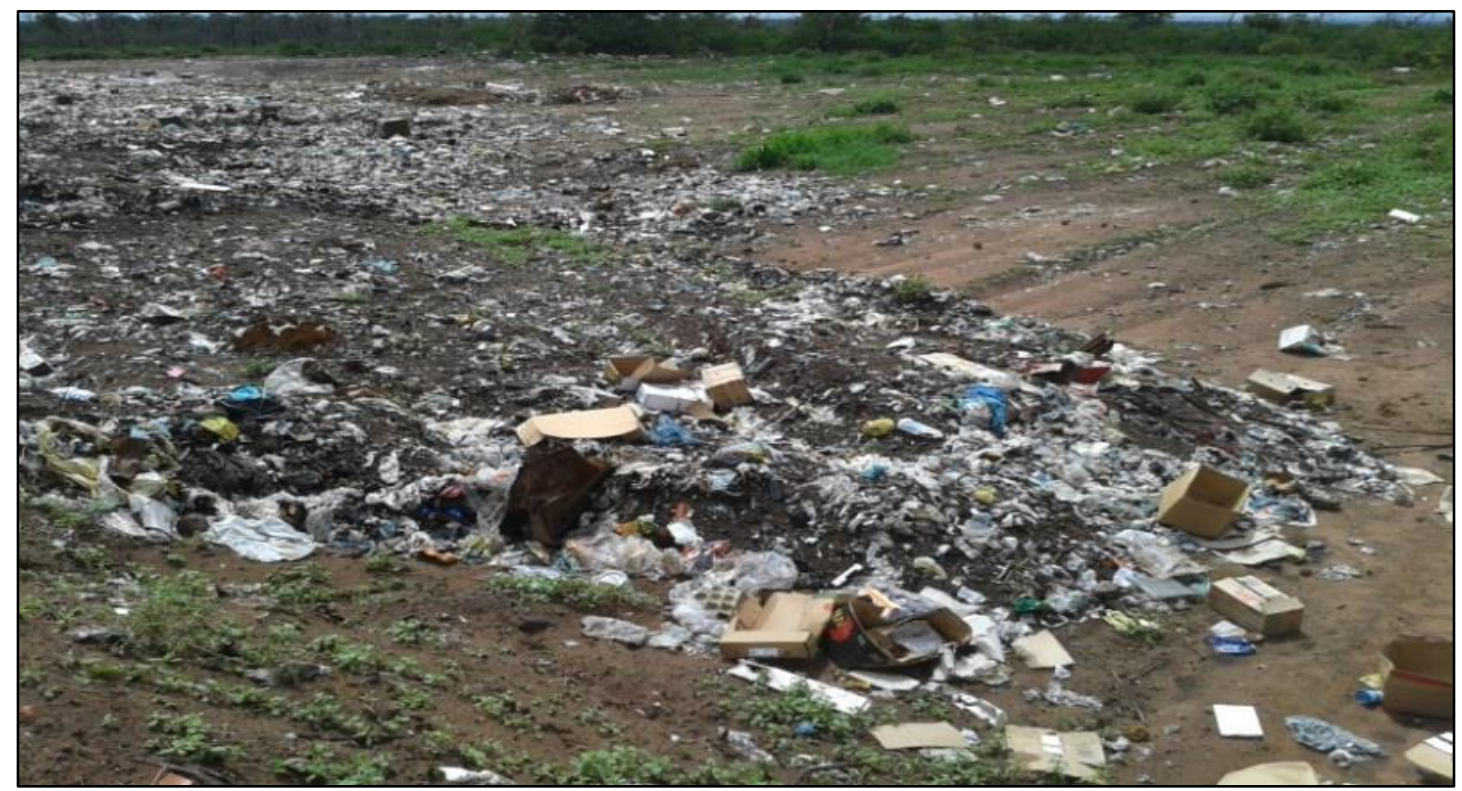

Figura 5: Acumulo dos resíduos sólidos sob a área do aterro controlado. Levantamento de Campo, 2015.

Identifica-se na (Figura 6) a exposição de substâncias e materiais nocivos, ao qual deveria ser separado do lixo comum e encaminhado para um tratamento adequado e posteriormente descartado em uma destinação adequada especifica. Tendo como obrigatoriedade de implantação da logística reversa da Lei $\mathrm{n}^{\mathrm{o}} 12.305$, estando presente como conceito moderno na Politica Nacional de Resíduos Sólidos.

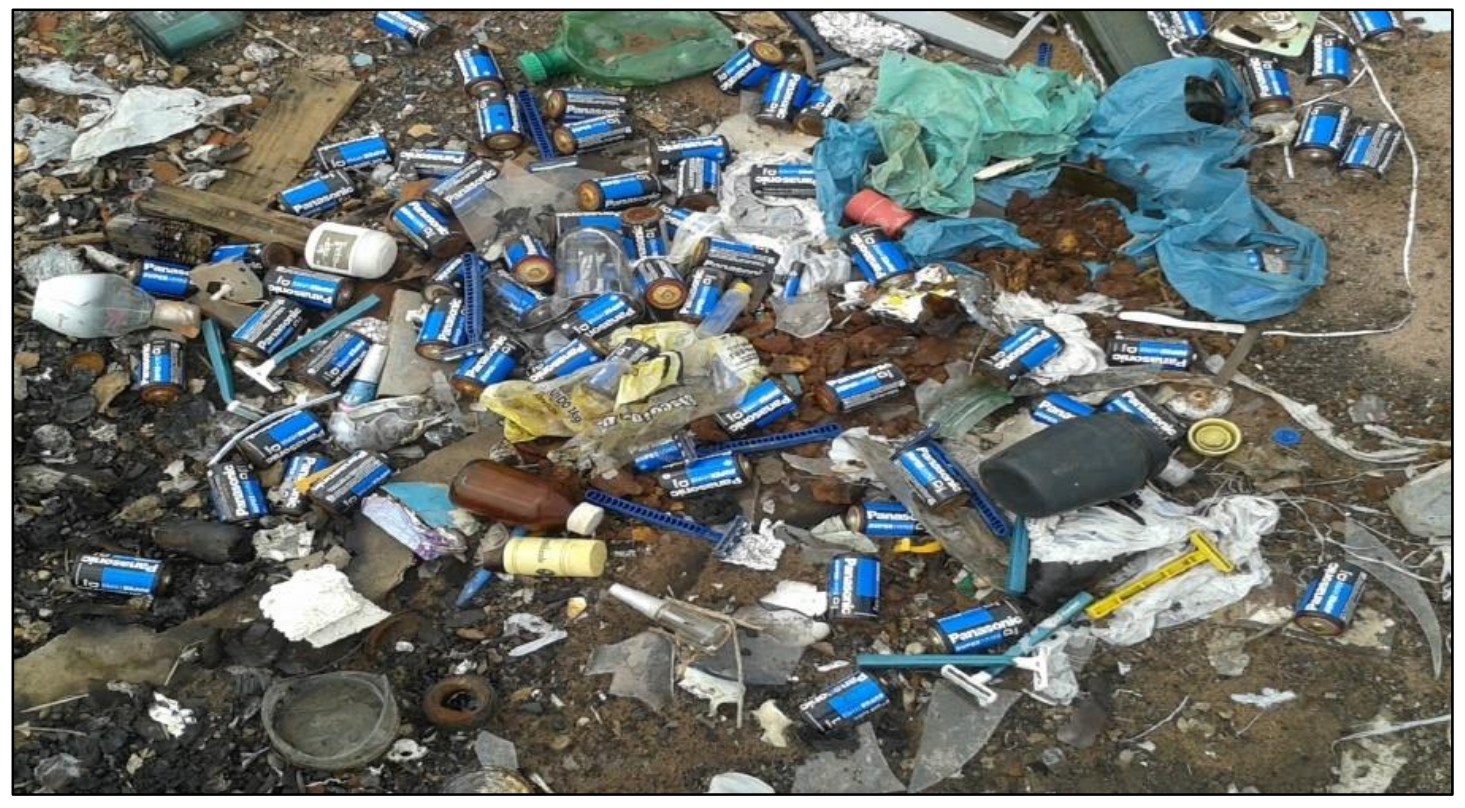

Figura 6: Substância nociva exposta sob o solo do aterro controlado. Levantamento de campo, 2015. 
Diante da inexistência do controle na disposição dos resíduos sólidos sob a área do aterro controlado alguns visam à utilização de um planejamento integral na gestão dos resíduos sólidos no qual se encontram em estado de deficiência na destinação final de tais resíduos, visando promover como subsidio para minimizar possíveis meios de contaminação do solo. Castilhos (2003) e Lima (2001) corroboram que nas ações de tratamento e disposição final dos resíduos sólidos, existe a necessidade de um planejamento que envolve tecnologia e engenharia para a implementação de aterros sanitários.

A identificação e monitoramento dos resíduos que são depositados na área podem ser realizados em visão de um controle que possa intervir na contaminação do solo, mediante diagnóstico detalhado visando os riscos que os resíduos podem causar e possivelmente uma investigação confirmatória dos possíveis fatores que alteram a qualidade do solo. Visando atribuir a um dos objetivos da Política Nacional de Resíduos Sólidos que trata no Art. $7^{\circ}$, inciso II sobre a não geração, redução, reutilização, reciclagem e tratamento dos resíduos sólidos, disposição final ambientalmente adequada dos rejeitos.

\section{CONCLUSÃO}

O atual aterro controlado não atende aos padrões de armazenamento de resíduos sólidos, no qual destacam-se inexistência de tratamento prévio e disposição irregular sem proteção do solo.

O recurso natural solo encontra-se em alta vulnerabilidade ambiental vinculada ao risco de processos de contaminação direta a alterações dos atributos químicos, físicos e biológicos. 


\section{REFERÊNCIAS}

BESEN, G. R. Coleta seletiva com inclusão de catadores: construção participativa de indicadores e índices de sustentabilidade. 2011. Tese (Doutorado) - Faculdade de Saúde Pública, Universidade de São Paulo, São Paulo, p.275.

BRASIL. Lei $\mathbf{N}^{\mathbf{0}}$ 12.305, de 2 de agosto de 2010. Institui a Política Nacional de Resíduos Sólidos; altera a Lei no 9.605, de 12 de fevereiro de 1998; e dá outrasprovidências. Disponível em: <http://www.planalto.gov.br/ccivil_03/_ato2007-2010/2010/lei/112305.htm>. Acesso em: 26 de novembro. 2015.

CASTILHOS JUNIOR, A. B. Resíduos Sólidos Urbanos: Aterro Sustentável para Municípios de Pequeno Porte. Florianópolis-SC, 2003. Editora Rima Artes e Textos.

FERNANDEZ VIÑA, M. B. D. Vertedoros Controlados de Resíduos Sólidos Urbanos: una Perspectiva Internacional. Universidad de Cantabria, Espanha. Setembro, 2000.

FUNASA. Fundação Nacional de Saúde. Manual de Saneamento. 1. ed. rev. Brasília, 2006, p.408.

INSTITUTO BRASILEIRO DE GEOGRAFIA E ESTATÍSTICA-IBGE. Censo demográfico 2010: sinopse. Rio de Janeiro: IBGE, 2010.

LIMA, J. D. Gestão de Resíduos Sólidos Urbanos no Brasil. Paraíba, 2001, Editora João Pessoa.

MELO, V. L. A. Estudos de referência para diagnóstico ambiental em aterros de resíduos sólidos. In: Congresso interamericano de engenharia sanitária e ambiental, 27, 2000. Anais... Fortaleza-CE, 2000.

MONTEIRO, J. H. P.; Figueiredo, C. E. M.; Magalhães, A. F.; Melo, M. A. F.; Brito, J. C. X.; Almeida, T. P. F.; Mansur, G. L. Manual de Gerenciamento Integrado de resíduos sólidos. Rio de Janeiro: IBAM, 2001.

PARIS, A. G. Redução das Emissões de gases de Efeito Estufa Listados no Protocolo de Quioto pelo Aproveitamento do Gás Gerado em Aterros Sanitários Utilizando Células a Combustível de Óxido Sólido. Universidade de São Paulo, São Paulo, 2007.

PMC - Prefeitura Municipal de Corrente. Plano municipal de coleta de resíduos sólidos, 2014. Corrente: PMC, 2014.

SISINNO, C. L. S.; Moreira, J. C. Avaliação da contaminação e poluição ambiental na área de influência do aterro controlado do Morro do Céu, Niterói, Brasil. Cadernos de Saúde Pública, v. 12, 1996.

SOBRINHO, N. L. C. Uma análise do balanço hídrico do aterro sanitário de Presidente Prudente. Viçosa/MG: Universidade Federal de Juiz de Fora, 2000.

TEIXEIRA, R. S. Caracterização e avaliação da contaminação do solo laterítico da área do depósito de resíduos sólidos urbanos de Londrina por metais pesados. Florianópolis/SC: Universidade Federal de Santa Catarina, 2008. 